\title{
Atomistic Insight into the Formation of Metal-Graphene One-Dimensional Contacts
}

Kretz, Bernhard; Pedersen, Christian Søndergaard; Stradi, Daniele; Brandbyge, Mads; Garcia-Lekue, Aran

Published in:

Physical Review Applied

Link to article, DOI:

10.1103/PhysRevApplied.10.024016

Publication date:

2018

Document Version

Publisher's PDF, also known as Version of record

Link back to DTU Orbit

Citation (APA):

Kretz, B., Pedersen, C. S., Stradi, D., Brandbyge, M., \& Garcia-Lekue, A. (2018). Atomistic Insight into the Formation of Metal-Graphene One-Dimensional Contacts. Physical Review Applied, 10(2).

https://doi.org/10.1103/PhysRevApplied.10.024016

\section{General rights}

Copyright and moral rights for the publications made accessible in the public portal are retained by the authors and/or other copyright owners and it is a condition of accessing publications that users recognise and abide by the legal requirements associated with these rights.

- Users may download and print one copy of any publication from the public portal for the purpose of private study or research.

- You may not further distribute the material or use it for any profit-making activity or commercial gain

- You may freely distribute the URL identifying the publication in the public portal 


\title{
Atomistic Insight into the Formation of Metal-Graphene One-Dimensional Contacts
}

\author{
Bernhard Kretz, ${ }^{1}$ Christian S. Pedersen, ${ }^{2}$ Daniele Stradi, ${ }^{2}$ Mads Brandbyge, ${ }^{2}$ and \\ Aran Garcia-Lekue ${ }^{1,3, *}$ \\ ${ }^{1}$ Donostia International Physics Center (DIPC), Paseo Manuel de Lardizabal 4, E-20018 San Sebastian, Spain \\ ${ }^{2}$ Center for Nanostructured Graphene (CNG), Department of Micro- and Nanotechnology (DTU Nanotech), \\ Technical University of Denmark, DK-2800, Kgs. Lyngby, Denmark \\ ${ }^{3}$ IKERBASQUE, Basque Foundation for Science, E-48013 Bilbao, Spain
}

(Received 16 February 2018; revised manuscript received 27 April 2018; published 14 August 2018)

\begin{abstract}
Motivated by the need to control the resistance of metal-graphene interfaces, we have simulated the structural and transport properties of edge contacts upon their formation. Our first-principles calculations reveal that the contacts evolve in a nontrivial way depending on the type of metal and the chemical contamination of the graphene edge. In particular, our results indicate that the origin of the low experimental resistance of chromium-graphene edge contacts is related to their weaker variation upon contamination and defect formation. In summary, by analyzing the distance dependence of the graphene-metal interaction and the relation between the reactivity and forces at the graphene edge, we shed new light on the mechanisms responsible for the diverse performance of experimentally fabricated graphene edge contacts.
\end{abstract}

DOI: 10.1103/PhysRevApplied.10.024016

\section{INTRODUCTION}

The idea of using graphene in electronic components, such as interconnects, comes naturally due to the material's small volume and excellent electrical and thermal properties $[1,2]$. However, many of the envisioned applications involve the formation of contacts between graphene and metallic electrodes [3-7], which will necessarily alter the electrical characteristics of graphene near the contact region [8-10]. Accordingly, huge efforts are being made to design metal-graphene (M-Gr) interfaces with minimal contact resistance [11-16].

Achieving a good contact performance has been proven difficult to achieve with conventional on-top contact schemes, in which the large overlap between the graphene $\pi$-system and the metal often yields a large contact resistance [17-21]. An alternative approach is to use edge contacts, where the graphene edge is directly connected to the metal, forming a one-dimensional (1D) metal-graphene interface [22]. Within this new contact scheme, the spatial overlap between the graphene and the metal is reduced to a minimum and, ideally, a covalent bond is formed between the metal atoms and the graphene. This leads to substantially lower resistance in edge contacts as compared to on-top contacts [22-28].

Despite their outstanding potential, understanding and controlling the transport properties of edge contacts remains a challenging task, mainly due to the difficulty

\footnotetext{
*wmbgalea@lg.ehu.es
}

of obtaining statistically reproducible M-Gr 1D contact geometries. Indeed, considerable device-to-device resistance variations have been observed $[15,22,26]$, which can be ascribed to the use of different fabrication strategies. For instance, before electrode deposition the graphene is often shaped by reactive ion or plasma etching. This technique is efficient in exposing graphene edges and removing residues $[15,22,25,29,30]$, but might also significantly alter the structural and chemical conformation of the contact.

A few first-principle calculations have already discussed the influence of chemical contamination by foreign atomic species in graphene edge contacts, but assuming chemically abrupt M-Gr interfaces at equilibrium bonding conditions [22,31]. Yet, the final properties of the contacts are most likely governed by electrochemical processes involving both graphene (clean or contaminated) and metal. Additionally, the chemical reactivity during the contact formation will also depend on the metal of choice. This complex atomic-scale scenario is not accessible experimentally, which indicates an urgent need for a systematic theoretical study.

In this work, we investigate the formation of $\mathrm{M}-\mathrm{Gr}$ edge contacts using different contaminants and metal electrodes. Special attention is paid to the reactivity of the metal and the graphene edges, a relevant question so far neglected in the study of this type of contact. Based on our first-principles calculations, we elucidate the complex dependence of the graphene edge-contacts resistance on both the termination chemistry of graphene and the metal work function $[32,33]$. 


\section{METHODS}

The electronic structures and geometries of 1D metalgraphene contacts upon their formation are calculated using density-functional theory (DFT), as implemented in the SIESTA code [34]. To describe the model graphene edge contact schematically depicted in Fig. 1(a), we use a supercell made up of a metallic slab containing five layers of $\mathrm{Ni}(111), \operatorname{Cr}(110), \mathrm{Ti}(0001)$, or $\mathrm{Au}(111)$, and a graphene sheet containing $40 \mathrm{C}$ rows. The graphenic subsystem is passivated with $\mathrm{H}$ at the edge furthest from the contact region, and unpassivated or functionalized with $\mathrm{F}, \mathrm{F}_{2}$, or $\mathrm{O}$ at the edge facing the metal surface. This choice of contaminants is based on stability calculations of different graphene edge functionalizations, performed using the VASP [35-38] code (for details see Ref. [39]).

In order to determine the relevant contact configurations, we first calculate the total system energy as a function of the separation distance between both subsystems (metal, and pristine or contaminated graphene sheet) at frozen geometries. This allows us to approximately estimate the minimum-energy and maximum-force separations, from where all the atoms of the graphenic subsystem (C, $\mathrm{H}$, and contaminant atoms) as well as the three topmost metal layers are allowed to relax (for more details about metal-graphene distances see Ref. [39]). The optimized interface geometries obtained in this way are referred to as minimum-energy and maximum-force contact conformations throughout the paper. It is worth mentioning that more realistic interface structures might be obtained using modern stochastic methods $[40,41]$. However, this would pose a challenging task that falls outside the scope of the present work. Thus, herein we focus on the optimized structures obtained by standard DFT, which might indeed serve as excellent starting points for future studies based on stochastic approaches.

Based on the relaxed contact geometries, we compute the elastic transmission with TRANSIESTA [42,43], which combines DFT with nonequilibrium Green's function methods. The scattering region is constructed by connecting the metal and graphene electrodes at the lefthand and right-hand sides of the contact region, aiming to prevent spurious effects related to the graphene channel length. Due to the asymmetric arrangement of the two electrodes, a buffer region of $16 \mathrm{C}$ atom rows is introduced. Regarding the termination of the graphene edge, it is found to have a minor influence on the resistance of the uncontaminated contacts (see Ref. [39]). Although the scenario is likely to change in the case of contaminated graphene edges, to allow direct comparisons between different contacts to the same metal, here we have decided to consider only zigzag-edged graphene both for uncontaminated and chemically modified cases. Moreover, since the metal contacts used experimentally are not crystalline and thus difficult to simulate, we have chosen one single

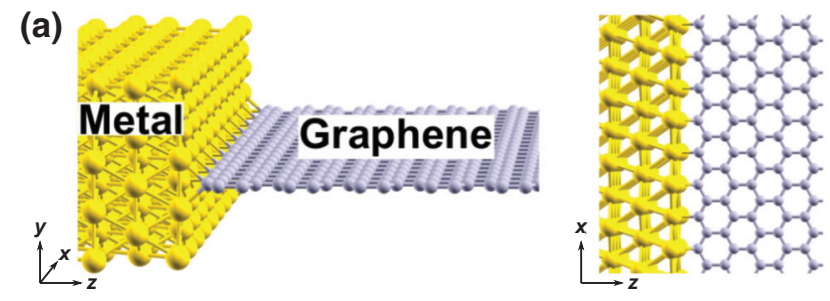

(b)

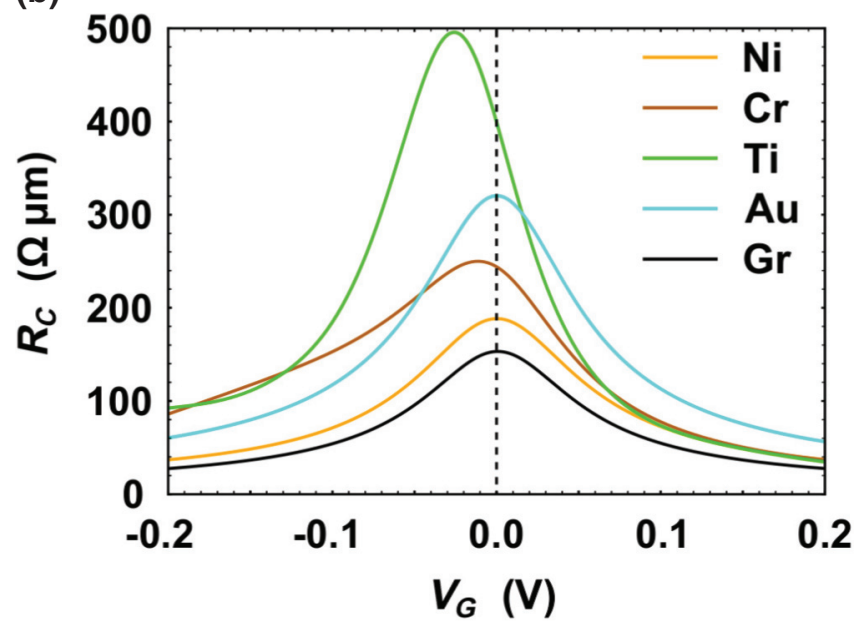

FIG. 1. (a) Side (left) and top (right) view of a model M-Gr edge contact. (b) Calculated contact resistance at $300 \mathrm{~K}$ as a function of gate voltage $\left(V_{G}\right)$ for different graphene edge contacts with different metals, namely Ni (orange), $\mathrm{Cr}$ (brown), Ti (brown), and $\mathrm{Au}$ (cyan). The intrinsic resistance of free-standing graphene is included as reference (black curve). A gate voltage of $V_{G}= \pm 0.2 \mathrm{~V}$ corresponds to a doping level of approximately $4 \times 10^{12} \mathrm{~cm}^{-2}$.

crystalline orientation for each of the metals considered. This approach allows us to focus on trends, making our results for different systems fairly comparable.

For all the calculations, a Monkhorst-Pack $k$-point grid with $23 \times 3 \times 1 k$-points is used for the Brillouin zone sampling and the mesh cutoff is set to $400 \mathrm{Ry}$. A double- $\zeta$ polarized basis set is utilized with 0.02 Ry energy shift to control the cutoff radii of the basis orbitals. The exchangecorrelation energy is approximated by the Perdew-BurkeErnzerhof (PBE) [44] flavor of the generalized gradient approximation (GGA). Structures are relaxed until forces are smaller than $0.01 \mathrm{eV} / \AA$. The transmission functions are sampled over $767 \times 5 \times 1 k$-points.

Based on the first-principles transport results, the effect of gate voltage is modeled by a rigid shift of the Fermi level. The thermally averaged resistance as a function of the gate voltage $\left(V_{G}\right)$ is then obtained from the calculated transmission curve $T(E)$ as follows [26]:

$$
\frac{1}{R_{C}}=\frac{G_{0}}{L} \int T(E) \frac{e^{\left(E-\left(E_{F}+e V_{G}\right)\right) / k_{B} T}}{\left(1+e^{\left(E-\left(E_{F}+e V_{G}\right)\right) / k_{B} T}\right)^{2}} \frac{d E}{k_{B} T},
$$


where $L$ is the transverse length of the contact along the periodic $x$ direction, $G_{0}$ is the quantum of conductance, and $T$ is the temperature.

\section{RESULTS AND DISCUSSION}

\section{A. Conductance properties of clean contacts}

To examine whether the wide range of resistance values reported for different metals can be attributed to their intrinsic different nature $[22,26]$, we first study clean M-Gr contacts at equilibrium. Figure 1(b) shows the calculated resistance of uncontaminated edge contacts to a representative set of metal surfaces [31], i.e., $\operatorname{Cr}(110), \operatorname{Ti}(0001)$, $\mathrm{Ni}(111)$, and $\mathrm{Au}(111)$. Unexpectedly, the maximum resistance is obtained when contacting graphene to Ti. In fact, the performance is even worse than with the more inert $\mathrm{Au}$ electrode. The origin of such poor electronic transmission across the Ti-Gr interface is the mismatch between the Fermi level states of the metal and those at the graphene edge, as was reported to occur for epitaxial graphene on $\mathrm{Ti}$ [45]. In any case, despite small variations, all four metals exhibit contact resistances of the same order of magnitude, within roughly a factor of two from the ideal contact resistance of graphene. Our calculated resistances are in qualitative agreement with those reported in Ref. [46], despite some numerical discrepancies which can be attributed to methodological issues (in particular, to the short channel length employed in their studies). Remarkably, this indicates that the experimental orders-of-magnitude disparity in the resistance of edge contacts can not be solely due to the use of different metal electrodes. For this reason, in the following we turn our attention to the influence of graphene's chemical termination.

\section{B. Interface reactivity of contaminated contacts}

The plasma employed to etch graphene is usually generated from a mixture of gases containing fluorine $(\mathrm{F})$ or oxygen $(\mathrm{O})[22,25,26,47]$ and, thus, we consider such elements as possible edge contaminants. According to our energetics considerations (see details in Ref. [39]), monoand difluorination [48], and mono-oxidation are found to be the most favorable chemical edge modifications. For each of these contaminant groups, we mimic the experimental fabrication process by investigating the contact formation upon approach of the metal to the graphenic system (see the details in the Methods section). Particular focus is placed on the maximum total attractive force conformation, as this corresponds to the separation at which metal and graphene will be most reactive.

Figure 2 shows the forces in the $z$ direction (perpendicular to the metal surface) on the atoms of the clean or contaminated graphene sheet, at a distance of maximum total attractive force. The following representative systems are studied: (i) three Ni-based contacts, composed of $\mathrm{Ni}(111)$ (a)

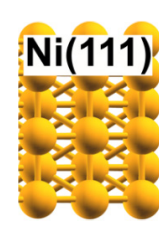

(c)

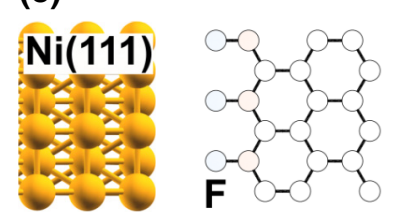

(b)

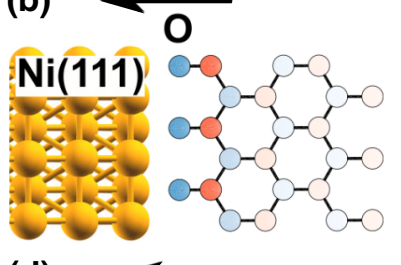

(d)

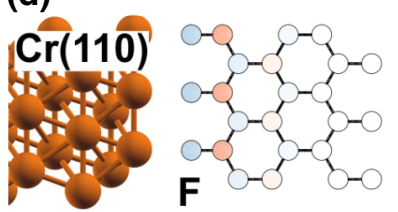

Forces on $\mathbf{C}$ atoms at interface

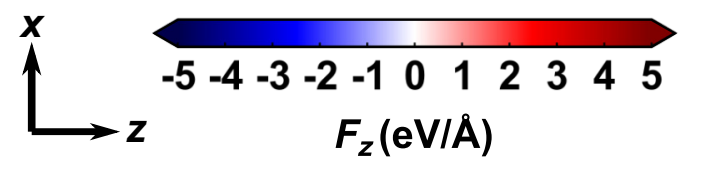

FIG. 2. Forces in the $z$ direction on the atoms of the graphenic subsystem, for four selected contacts at maximum-force conformation. Blue (red) shades indicate forces pointing towards (away from) the metal surface. The forces for the following contacts are shown: (a) Ni-Gr, (b) Ni-O-Gr, (c) Ni-F-Gr, and (d) Cr-F-Gr.

and clean, O-contaminated, or F-contaminated graphene (Ni-Gr, Ni-O-Gr, and Ni-F-Gr, respectively); (ii) a Crbased contact composed of $\mathrm{Cr}(110)$ and F-contaminated graphene (Cr-F-Gr). Blue (red) shades indicate forces on the individual atoms pointing towards (away from) the metal surface. To allow direct comparisons, the forces for the contaminated graphene contacts are rescaled with respect to those for clean graphene. The arrows in Fig. 2 indicate the magnitude and direction of the forces on the atoms closest to the metal, i.e., on the first $\mathrm{C}$ row for the clean $\mathrm{Ni}$-graphene contact, and on the $\mathrm{F}$ or $\mathrm{O}$ atoms for the contaminated contacts. In all cases, the net forces on the edges of the graphenic subsystems are pointing towards the metal surface, i.e., graphene feels an attractive force exerted by the metal. We see how the magnitude of the force strongly depends both on the chemical termination of graphene as well as on the metal of choice. For the Ni electrode we find that the attraction is maximum for the clean metal-graphene interface, the attractive force always being reduced upon contamination. However, the degree to which the force is quenched depends on the type of contaminant: for the oxidized case the forces remain quite large [Fig. 2(b)], while in the fluorinated case they become extremely small [Fig. 2(c)]. Interestingly, if one substitutes $\mathrm{Ni}$ by $\mathrm{Cr}$, the force on the fluorinated graphene edge increases significantly [Fig. 2(d)], while it remains almost constant upon contamination with O (see Fig. S7 in [39]).

In addition, we investigate to which degree the interface evolution depends on the specific contacting site between the metal and the graphenic system. With this aim, we 
(a)

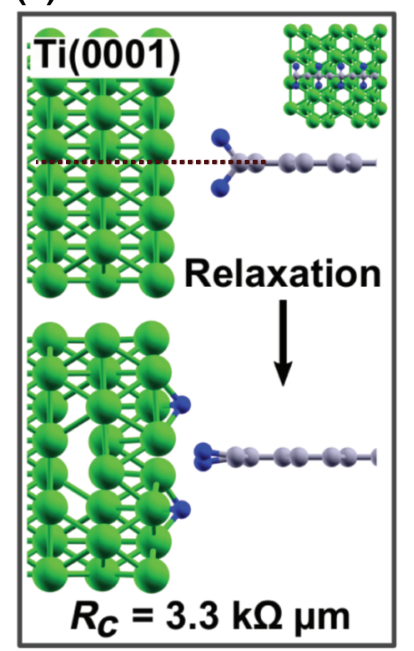

(b)

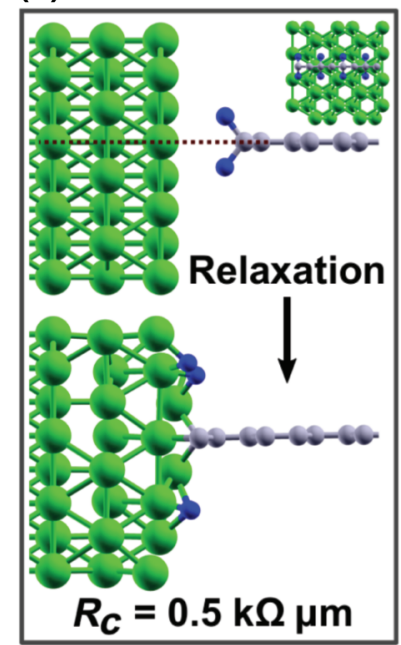

FIG. 3. (a),(b) Side and top views of two Ti-F - Gr interfaces with different contacting sites (marked with horizontal dashed lines). Top panels: maximum-force conformations before structural optimization. Lower panels: contact geometry upon relaxation, together with the corresponding resistance at $T=300 \mathrm{~K}$ and $V_{G}=0 \mathrm{~V}$.

consider a $\mathrm{Ti}(0001)$ electrode and a difluorinated graphene sheet, which are brought into contact at two different relative positions, as shown in Fig. 3. Both systems are fully relaxed from their maximum-force conformation (upper figures) and, interestingly, the equilibrium structures are remarkably different (lower figures). For the initial contact configuration of Fig. 3(a), only one of the F atoms gets stripped off from the graphene edge and bonds to the metal. For the contact geometry in Fig. 3(b) instead, both $\mathrm{F}$ atoms are removed from the edge and adsorbed onto the metal substrate. In the latter case, the graphene eventually forms a clean contact to $\mathrm{Ti}$ and the contact resistance is thus significantly reduced.

\section{Resistance characteristics of $1 D$ contacts}

The results presented in the previous section clearly indicate that an appropriate interpretation of experimental results requires more realistic considerations of the complex interplay between the interface structure and its reactivity. Accordingly, we carry out a systematic analysis of the structural and chemical changes of the 1D contacts upon their formation, for all the possible combinations of metal electrodes ( $\mathrm{Au}, \mathrm{Ni}, \mathrm{Cr}$, and $\mathrm{Ti}$ ) and graphene terminations (clean, and $\mathrm{F}-, \mathrm{F}_{2^{-}}$, or O-contaminated) considered in this work. We find that, when transition metals are used, the final contacts always fall in one of the three conformations schematically depicted in Fig. 4(a). Thus, the $F_{2}$ modification of the graphene edge, despite being energetically more favorable for isolated graphene [48],

(a)

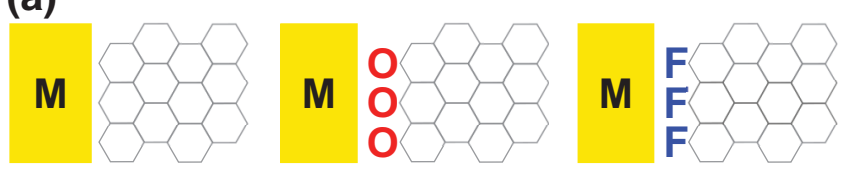

(b)

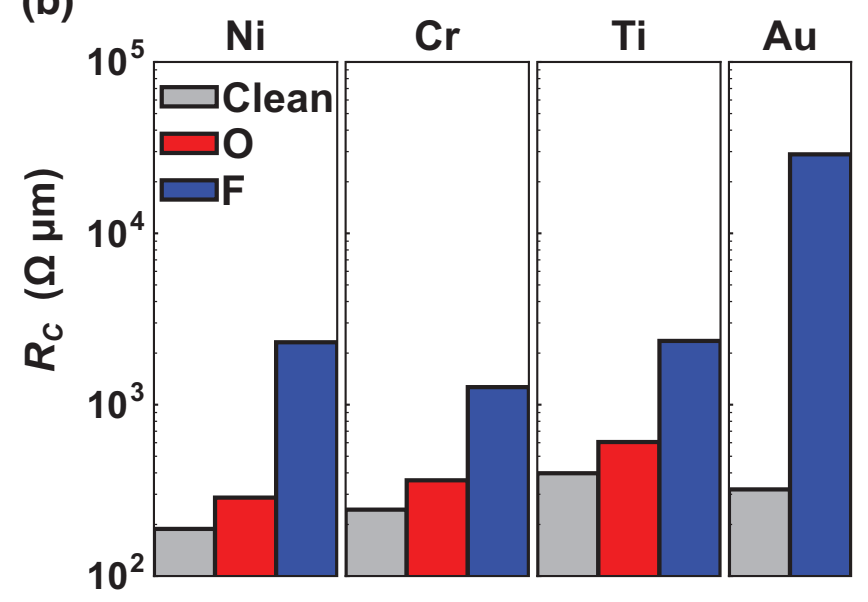

FIG. 4. (a) Schematic illustration of the three equilibrium conformations for transition-metal-based contacts. (b) Histogram of the contact resistance at $T=300 \mathrm{~K}$ and $V_{G}=0 \mathrm{~V}$, as obtained for different metals $(\mathrm{Ni}, \mathrm{Cr}, \mathrm{Ti}$, and $\mathrm{Au})$ and graphene edge contaminations $(\mathrm{O}, \mathrm{F})$.

is not likely to occur under the influence of transitionmetal electrodes in edge-contacted topology. When $\mathrm{Au}$ is used, instead, the graphene edges (clean or contaminated) are not modified by the interaction with the metal. The reason behind this is the low reactivity of $\mathrm{Au}$ and the subsequent weak interplay between the metal and the graphene edge, which might also explain the bad performance of experimentally fabricated Au-based 1D contacts [22].

Having determined the structural and chemical conformation of the 1D contacts, we now focus on their conductance properties. The calculated zero gate voltage resistances of the different interfaces studied here are compiled in Fig. 4(b). From our results, it is clear that, regardless of the metal, the presence of contaminants reduces the efficiency of electron transmission across the contacts. However, the degree to which the contamination reduces the conductance and, thus, deteriorates the contact performance depends on the chemical species at the graphene edge. Oxygen contamination does not significantly alter the contact resistance for $\mathrm{Ni}, \mathrm{Cr}$, and $\mathrm{Ti}$. In contrast, when graphene is terminated with fluorine, the resistance is always drastically enlarged (by at least an order of magnitude). There are two intertwined factors that play a role in this increase of contact resistance upon $\mathrm{F}$ contamination. The first is that $\mathrm{F}$ can only form one covalent bond and, therefore, the contact between the metal electrode and the graphene edge is quite weak. The second is 
(a)

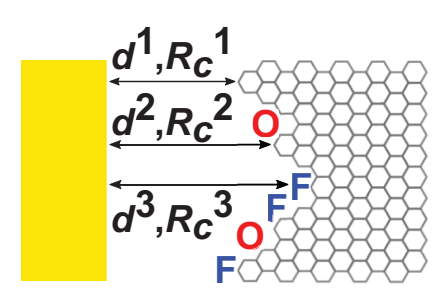

(b)

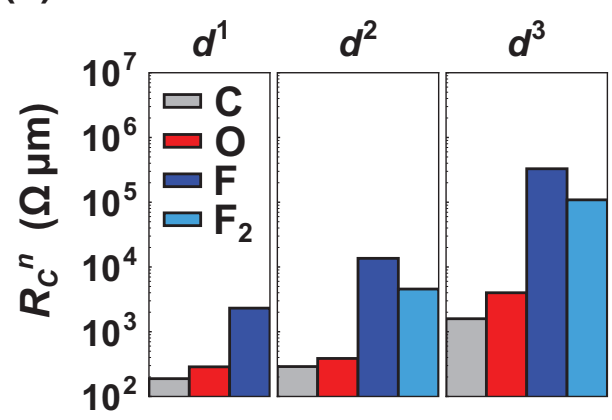

(c)

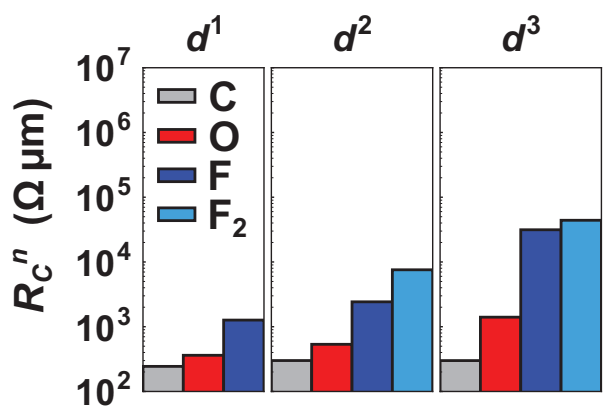

FIG. 5. (a) Schematic model of a corrugated metal-graphene 1D contact. The distances $d^{1}, d^{2}$, and $d^{3}$ denote metal-graphene distances at minimum energy, maximum force, and maximum force distance plus $1 \AA$, respectively. The corresponding resistances are denoted by $R_{C}^{1}, R_{C}^{2}$, and $R_{C}^{3}$. (b),(c) show the histograms of contact resistance at $T=300 \mathrm{~K}$ and $V_{G}=0 \mathrm{~V}$ for the clean (gray), O- (red), $\mathrm{F}$ - (blue), and $\mathrm{F}_{2}$ - (light blue) contaminated $\mathrm{Ni}$ and $\mathrm{Cr}$ electrodes.

the larger separation distance between the metal and the Fcontaminated graphene edge as compared to the clean one (see Table S1 in [39]). As expected from its inert character, these two effects are magnified for $\mathrm{Au}$, giving rise to a contact resistance more than an order of magnitude larger than for other metals.

The contacts employed in our simulations, made up of perfect crystalline metals and graphene with straight edges, are highly idealized structures. Experimental contacts will deviate in different ways from these theoretical systems, e.g., structural defects are expected and chemical contamination is most likely not uniform. Figure 5(a) displays a schematic model of a corrugated metal-graphene junction, which contains an irregular graphene edge with inhomogeneous fluorine and oxygen contamination. In this model, we consider the following representative distances from the metal to the graphene edge (clean or contaminated): $d^{1}$ (distance of minimum energy), $d^{2}$ (distance of maximum force), and $d^{3}$ (distance of maximum force plus $1 \AA$ ). Taking this model as a reference, and using $\mathrm{Ni}$ and $\mathrm{Cr}$ as representative metals, transport calculations for each distance and different graphene edge terminations (clean, oxidized, mono- or difluorinated) are carried out separately. The realistic system can be viewed as a combination of the different simulated scenarios and, thus, we can extract a range of values for the resistance of the model contact in Fig. 5(a) from the calculations reported in the following.

Figures 5(b) and 5(c) show the zero-gate-voltage resistance for clean, oxidized, and mono- or difluorinated graphene sheets at different distances from the $\mathrm{Ni}$ and $\mathrm{Cr}$ metal electrodes (additional results in [39]). As expected, the resistance rises as the graphenic subsystem gets further from the metal. However, the degree at which the quality of the contact is deteriorated depends on the type of metal. For Ni contacts, the resistance smoothly increases as the graphene sheet is moved further away. The increase is more pronounced for contaminated interfaces, particularly for fluorinated ones. At $d^{3}$, the resistance of Ni-F-Gr is more than two orders of magnitude larger than at the equilibrium distance.

Interestingly, the situation is quite different for $\mathrm{Cr}(110)$. When connected to clean graphene, we find that the contact resistance does not depend much on the Cr-Gr separation distance. This behavior can be ascribed to the relatively short bond distance, both at minimum-energy and maximum-force conformations. Furthermore, when the graphene edge is contaminated, either with $\mathrm{F}$ or $\mathrm{O}$, increasing the distance to the $\mathrm{Cr}$ electrode yields a lower relative variation of the resistance as compared to $\mathrm{Ni}$ [31]. In other words, the quality of Cr-based contacts is less damaged upon contamination. We can thus conclude that $\mathrm{Cr}$ is the best candidate to form 1D graphene contacts, in agreement with the experimental observations reported by Wang et al. [22].

\section{Spin polarization at contaminated interfaces}

Finally, the spin-polarization character of the electron transport across 1D M-Gr contacts is addressed, using CrF-Gr and Ni-F-Gr as illustrative systems. Figure 6 shows the spin-resolved density of states projected onto the interface atoms of a Cr-F-Gr contact, at the representative separation distances described above. At the distance of minimum energy $\left(d^{1}\right)$, the coupling to the metal destroys the spin-polarized attributes of the graphene zigzag edge, and the corresponding projected density of states (PDOS) exhibits spin-unpolarized behavior. As the graphenic subsystem is detached from the Cr surface, the interaction with the metal is weakened and a net spin polarization rapidly builds up. Indeed, at $d^{3}$ the PDOS on the first C row is comparable to that for isolated hydrogen-passivated zigzagedged graphene. In the case of Ni-F-Gr, instead the electronic properties exhibit strong spin-polarization character at any separation distance, which can be attributed to the intrinsic spin-polarized nature of Ni (see Fig. S10 in [39]). 


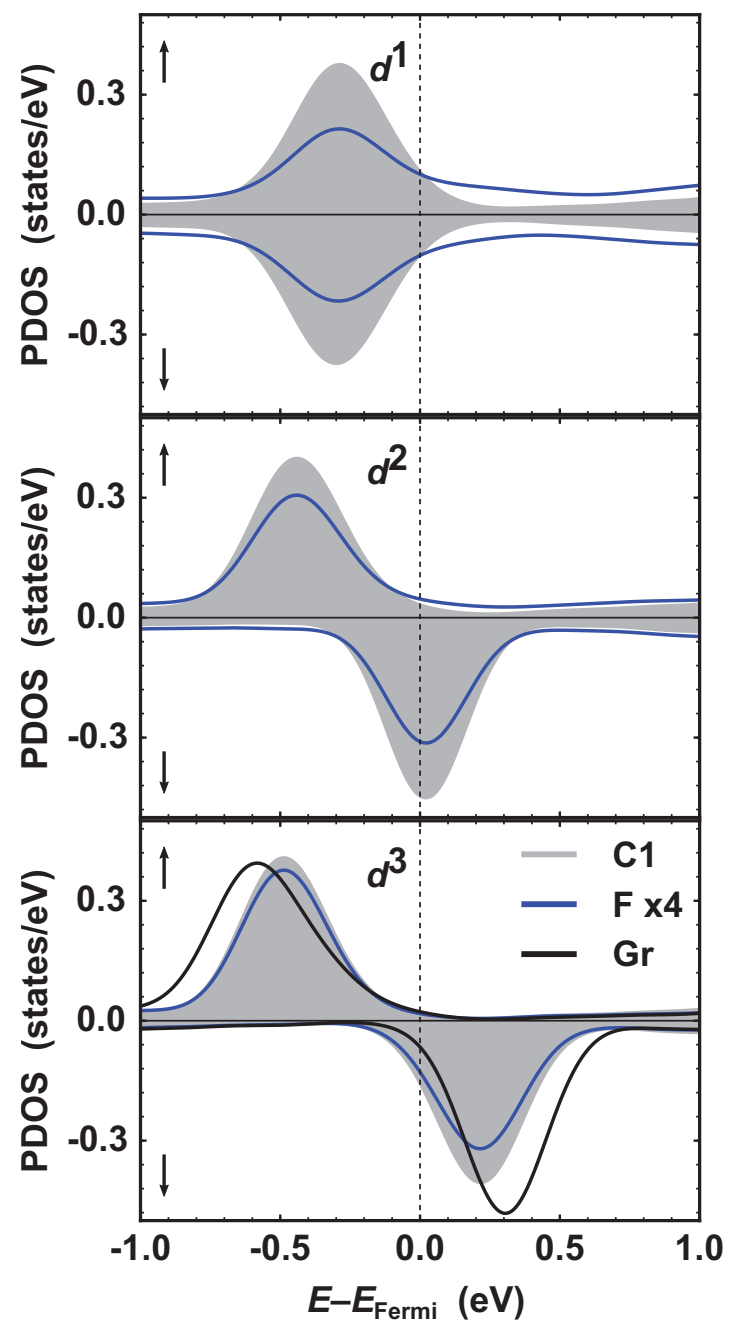

FIG. 6. Spin-up $(\uparrow)$ and spin-down $(\downarrow)$ calculated density of states projected onto the interface atoms of the Cr-F-Gr contact, at distances $d^{1}$ (top panel), $d^{2}$ (middle panel), and $d^{3}$ (bottom panel). In the bottom panel the corresponding PDOS of a graphene zigzag edge is shown for comparison. For visualization purposes, the PDOS of F has been multiplied by 4 .

The spin-polarization properties studied above will eventually manifest in the conductance across the contacts. In fact, as shown in Table I, the Ni-based contact exhibits a large spin-filtering efficiency at any separation distance. In contrast, when $\mathrm{Cr}$ is used as electrode material, the spin polarization of the conductance predicted at large separation distances is completely quenched when the graphene is bound to the metal. Hence, the spinpolarization properties of the contact might be greatly modified both by contamination and by the use of different metallic electrodes.

\section{CONCLUSIONS}

In summary, we investigate the role of atomic-scale chemical reactivity on the formation and resistance
TABLE I. Spin-filtering efficiency, defined as $(G \uparrow-G \downarrow) /$ $(G \uparrow+G \downarrow)$, at zero gate voltage and different separation distances.

\begin{tabular}{lccc}
\hline \hline & \multicolumn{3}{c}{ Spin-filtering efficiency \% } \\
\cline { 2 - 4 } & $d^{1}$ & $d^{2}$ & $d^{3}$ \\
\hline Ni-F-Gr & -74.4 & -89.0 & -92.8 \\
Cr-F-Gr & -0.4 & -32.5 & -86.1 \\
\hline \hline
\end{tabular}

performance of metal-graphene 1D contacts. We demonstrate that the final interface configuration depends on a delicate interplay between the type of metal, the contamination of the graphene edge, and the contacting site. Moreover, structural defects and chemical inhomogeneities are found to induce significant changes in the conductance properties and even the appearance of spin-filtering effects. Interestingly, we find that Cr-based contacts exhibit the lowest dependence on contamination and structural irregularities, which explains why $1 \mathrm{D}$ contacts fabricated using $\mathrm{Cr}$ electrodes have the lowest interface resistance.

All in all, our results allow us to conclude that the use of different metals and etching agents is crucial to produce $1 \mathrm{D}$ contacts with resistance values suitable for their use in real applications. Of course, the actual resistance of a 1D M-Gr contact depends on the density and distribution of defects and contaminants, which in turn is conditioned by the exact fabrication process. This kind of information is very difficult to extract experimentally and, thus, a quantitative assessment of measured resistances is out of the scope of this work. Nevertheless, our atomistic simulations are very valuable for understanding the origin of the device-to-device variations and, more importantly, might be helpful for guiding the future design of metal-graphene contacts.

\section{ACKNOWLEDGMENTS}

This work was supported by the Spanish Ministerio de Economía y Competitividad (MINECO) (Grant No. MAT2016-78293-C6-4-R) and the Basque Dep. de Educación and UPV/EHU (Grants No. IT-756-13 and PI-20161-0027). The Center for Nanostructured Graphene (CNG) is sponsored by the Danish Research Foundation, Project No. DNRF103. D.S. acknowledges support from the HCØ DTU-COFUND program. We thank Dr. B. S. Jessen for useful comments on our manuscript and Prof. P. Bøggild for helpful discussions.

[1] A. H. Castro Neto, F. Guinea, N. M. R. Peres, K. S. Novoselov, and A. K. Geim, The electronic properties of graphene, Rev. Mod. Phys. 81, 109 (2009).

[2] Alexander A. Balandin, Suchismita Ghosh, Wenzhong Bao, Irene Calizo, Desalegne Teweldebrhan, Feng Miao, and 
Chun Ning Lau, Superior thermal conductivity of singlelayer graphene, Nano Lett. 8, 902 (2008).

[3] Y.-M. Lin, C. Dimitrakopoulos, K. A. Jenkins, D. B. Farmer, H.-Y. Chiu, A. Grill, and Ph. Avouris, 100-GHz transistors from wafer-scale epitaxial graphene, Science 327, 662 (2010).

[4] Yanqing Wu, Keith A. Jenkins, Alberto Valdes-Garcia, Damon B. Farmer, Yu Zhu, Ageeth A. Bol, Christos Dimitrakopoulos, Wenjuan Zhu, Fengnian Xia, Phaedon Avouris, and Yu-Ming Lin, State-of-the-art graphene highfrequency electronics, Nano Lett. 12, 3062 (2012).

[5] Thanasis Georgiou, et al., Vertical field-effect transistor based on graphene-WS2 heterostructures for flexible and transparent electronics, Nat. Nanotechnol. 8, 100 (2013).

[6] Ten years in two dimensions, Editorial, Nat. Nanotechnol. 9, 725 (2014)

[7] Le Huang, Huilong $\mathrm{Xu}$, Zhiyong Zhang, Chengying Chen, Jianhua Jiang, Xiaomeng Ma, Bingyan Chen, Zishen Li, Hua Zhong, and Lian-Mao Peng, Graphene/Si CMOS hybrid hall integrated circuits, Sci. Rep. 4, 5548 (2014).

[8] G. Giovannetti, P. A. Khomyakov, G. Brocks, V. M. Karpan, J. van den Brink, and P. J. Kelly, Doping Graphene with Metal Contacts, Phys. Rev. Lett. 101, 026803 (2008).

[9] Salvador Barraza-Lopez, Mihajlo Vanević, Markus Kindermann, and M. Y. Chou, Effects of Metallic Contacts on Electron Transport through Graphene, Phys. Rev. Lett. 104, 076807 (2010).

[10] Filippo Giubileo and Antonio Di Bartolomeo, The role of contact resistance in graphene field-effect devices, Prog. Surf. Sci. 92, 143 (2017).

[11] A. D. Franklin, S. J. Han, A. A. Bol, and W. Haensch, Effects of nanoscale contacts to graphene, IEEE Electr. Device Lett. 32, 1035 (2011).

[12] A. D. Franklin, S. J. Han, A. A. Bol, and V. Perebeinos, Double contacts for improved performance of graphene transistors, IEEE Electr. Device Lett. 33, 17 (2012).

[13] J. S. Moon, et al., and P. Asbeck, Ultra-low resistance ohmic contacts in graphene field effect transistors, Appl. Phys. Lett. 100, 203512 (2012).

[14] Seung Min Song, Jong Kyung Park, One Jae Sul, and Byung Jin Cho, Determination of work function of graphene under a metal electrode and its role in contact resistance, Nano Lett. 12, 3887 (2012).

[15] Seung Min Song, Taek Yong Kim, One Jae Sul, Woo Cheol Shin, and Byung Jin Cho, Improvement of graphene-metal contact resistance by introducing edge contacts at graphene under metal, Appl. Phys. Lett. 104, 183506 (2014).

[16] Hua Zhong, Zhiyong Zhang, Bingyan Chen, Haitao Xu, Dangming Yu, Le Huang, and Lianmao Peng, Realization of low contact resistance close to theoretical limit in graphene transistors, Nano Res. 8, 1669 (2015).

[17] Fengnian Xia, Vasili Perebeinos, Yu-ming Lin, Yanqing $\mathrm{Wu}$, and Phaedon Avouris, The origins and limits of metal-graphene junction resistance, Nat. Nanotechnol. 6, 179 (2011).

[18] Seung Min Song and Byung Jin Cho, Contact resistance in graphene channel transistors, Carbonletters 14, 162 (2013).

[19] Bo Ma, Cheng Gong, Yanwei Wen, Rong Chen, Kyeongjae Cho, and Bin Shan, Modulation of contact resistance between metal and graphene by controlling the graphene edge, contact area, and point defects: An ab initio study, J. Appl. Phys. 115, 183708 (2014).

[20] Kurt Stokbro, Mads Engelund, and Anders Blom, Atomicscale model for the contact resistance of the nickelgraphene interface, Phys. Rev. B 85, 165442 (2012).

[21] Teresa Cusati, Gianluca Fiori, Amit Gahoi, Vikram Passi, Max C. Lemme, Alessandro Fortunelli, and Giuseppe Iannaccone, Electrical properties of graphene-metal contacts, Sci. Rep. 7, 5109 (2017).

[22] L. Wang, et al., One-dimensional electrical contact to a two-dimensional material, Science 342, 614 (2013).

[23] Joshua T. Smith, Aaron D. Franklin, Damon B. Farmer, and Christos D. Dimitrakopoulos, Reducing contact resistance in graphene devices through contact area patterning, ACS Nano 7, 3661 (2013).

[24] Tao Chu and Zhihong Chen, Understanding the electrical impact of edge contacts in few-layer graphene, ACS Nano 8, 3584 (2014).

[25] D. W. Yue, C. H. Ra, X. C. Liu, D. Y. Lee, and W. J. Yoo, Edge contacts of graphene formed by using a controlled plasma treatment, Nanoscale 7, 825 (2015).

[26] Khoong Hong Khoo, Wei Sun Leong, John T. L. Thong, and Su Ying Quek, Origin of contact resistance at ferromagnetic metal-graphene interfaces, ACS Nano 10, 11219 (2016).

[27] Hyung-Youl Park, et al., Extremely low contact resistance on graphene through n-type doping and edge contact design, Adv. Mater. 28, 864 (2016).

[28] Cheng Gong, Chenxi Zhang, Young Jun Oh, Weichao Wang, Geunsik Lee, Bin Shan, Robert M. Wallace, and Kyeongjae Cho, Electronic transport across metal-graphene edge contact, 2D Materials 4, 025033 (2017).

[29] Joshua A. Robinson, Michael LaBella, Mike Zhu, Matt Hollander, Richard Kasarda, Zachary Hughes, Kathleen Trumbull, Randal Cavalero, and David Snyder, Contacting graphene, Appl. Phys. Lett. 98, 053103 (2011).

[30] Min Sup Choi, Seung Hwan Lee, and Won Jong Yoo, Plasma treatments to improvemetal contacts in graphene field effect transistor, J. Appl. Phys. 110, 073305 (2011).

[31] Qun Gao and Jing Guo, Role of chemical termination in edge contact to graphene, APL Mater. 2, 056105 (2014).

[32] J. J. Palacios, A. J. Pérez-Jiménez, E. Louis, E. SanFabián, and J. A. Vergés, First-principles Phase-coherent Transport in Metallic Nanotubes with Realistic Contacts, Phys. Rev. Lett. 90, 106801 (2003).

[33] Johannes Svensson and Eleanor E. B. Campbell, Schottky barriers in carbon nanotube-metal contacts, J. Appl. Phys. 110, 111101 (2011).

[34] José M. Soler, Emilio Artacho, Julian D. Gale, Alberto García, Javier Junquera, Pablo Ordejón, and Daniel SánchezPortal, The SIESTA method for ab initio order-N materials simulation, J. Phys.: Condens. Matter 14, 2745 (2002).

[35] G. Kresse and J. Hafner, Ab initio molecular dynamics for liquid metals, Phys. Rev. B 47, 558 (1993).

[36] G. Kresse and J. Hafner, Ab initio molecular-dynamics simulation of the liquid-metal-amorphous-semiconductor transition in germanium, Phys. Rev. B 49, 14251 (1994).

[37] G. Kresse and J. Furthmüller, Efficiency of ab-initio total energy calculations for metals and semiconductors using a plane-wave basis set, Comput. Mater. Sci. 6, 15 (1996). 
[38] G. Kresse and J. Furthmüller, Efficient iterative schemes for $\mathrm{ab}$ initio total-energy calculations using a plane-wave basis set, Phys. Rev. B 54, 11169 (1996).

[39] See Supplemental Material at http://link.aps.org/supple mental/10.1103/PhysRevApplied.10.024016 for more data on metal-graphene distances, forces on interface atoms, contact resistances, spin polarization in a contaminated contact, energetics of contaminated graphene edges, influence of the termination of the graphene edge, and the electrostatic potential of a clean nickel-graphene contact.

[40] Georg Schusteritsch and Chris J. Pickard, Predicting interface structures: From $\mathrm{SrTiO}_{3}$ to graphene, Phys. Rev. B 90, 035424 (2014).

[41] Georg Schusteritsch, Steven P. Hepplestone, and Chris J. Pickard, First-principles structure determination of interface materials: The $\mathrm{Ni}_{x}$ InAs nickelides, Phys. Rev. B 92, 054105 (2015).

[42] Mads Brandbyge, José-Luis Mozos, Pablo Ordejón, Jeremy Taylor, and Kurt Stokbro, Density-functional method for nonequilibrium electron transport, Phys. Rev. B 65, 165401 (2002).

[43] Nick Papior, Nicolás Lorente, Thomas Frederiksen, Alberto García, and Mads Brandbyge, Improvements on non- equilibrium and transport Green function techniques: The next-generation transiesta, Comput. Phys. Commun. 212, 8 (2017).

[44] J. P. Perdew, K. Burke, and M. Ernzerhof, Generalized Gradient Approximation Made Simple, Phys. Rev. Lett. 77, 3865 (1996).

[45] Marcelo A. Kuroda, J. Tersoff, Dennis M. Newns, and Glenn J. Martyna, Conductance through multilayer graphene films, Nano Lett. 11, 3629 (2011).

[46] Yuki Matsuda, Wei-Qiao Deng, and William A. Goddard, Contact resistance for end-contacted metal-graphene and metal-nanotube interfaces from quantum mechanics, J. Phys. Chem. C 114, 17845 (2010).

[47] Filippo Pizzocchero, Lene Gammelgaard, Bjarke S. Jessen, José M. Caridad, Lei Wang, James Hone, Peter Bøggild, and Timothy J. Booth, The hot pick-up technique for batch assembly of van der Waals heterostructures, Nat. Commun. 7, 11894 (2016).

[48] Sumanta Bhandary, Gabriele Penazzi, Jonas Fransson, Thomas Frauenheim, Olle Eriksson, and Biplab Sanyal, Controlling electronic structure and transport properties of zigzag graphene nanoribbons by edge functionalization with fluorine, J. Phys. Chem. C 119, 21227 (2015). 